\title{
Revista Colombiana de

\section{De la insuficiencia cardiaca a la diabetes. ¿Existe un mecanismo común?}

\author{
María Juliana Rodríguez-González ${ }^{\mathrm{a}, *}$ y Johana Contreras ${ }^{\mathrm{b}}$ \\ a Programa de Falla Cardíaca y Trasplante de la Fundación Cardioinfantil-Instituto de Cardiología, Bogotá, Colombia \\ b Programa de Falla Cardíaca y Trasplante, Mount Sinai, Nueva York, Estados Unidos
}

Recibido el 9 de diciembre de 2019; aceptado el 17 de diciembre de 2019

\section{PALABRAS CLAVE \\ Diabetes; \\ Falla cardiaca; \\ Tratamiento}

\section{KEYWORDS}

Diabetes;

Heart failure;

Treatment
Resumen La falla cardíaca es una entidad de alta prevalencia, que se asocia con varias comorbilidades. Una de las asociaciones que ha generado alarma creciente es la diabetes mellitus. Al respecto, se afirma que hay una relación bidireccional entre estas dos entidades y se han estudiado múltiples hipótesis entre las cuales ha generado mayor aceptación la activación de la respuesta neurohumoral con hiperactivación del sistema nervioso simpático y posterior aparición de resistencia a la insulina.

( 2019 Publicado por Elsevier España, S.L.U. en nombre de Sociedad Colombiana de Cardiología y Cirugía Cardiovascular. Este es un artículo Open Access bajo la licencia CC BY-NC-ND (http:// creativecommons.org/licenses/by-nc-nd/4.0/).

\section{From cardiac failure to diabetes: Is there a common mechanism?}

Abstract Heart failure is a highly prevalent condition that is associated to various comorbidities. Diabetes mellitus is one of the associations that has generated increasing alarm. In this regard, it has been shown that there is a bidirectional relationship between these two conditions. Many hypotheses have been studied, among which the activation of the neurohumoral response with hyper-activation of the sympathetic nervous system and the subsequent appearance of insulin resistance, has been more widely accepted.

(c) 2019 Published by Elsevier España, S.L.U. on behalf of Sociedad Colombiana de Cardiología y Cirugía Cardiovascular. This is an open access article under the CC BY-NC-ND license (http:// creativecommons.org/licenses/by-nc-nd/4.0/).

\footnotetext{
* Autor para correspondencia.

Correo electrónico: mjrodriguez@cardioinfantil.org (M.J. Rodríguez-González).
}

La insuficiencia cardiaca y la diabetes mellitus tipo 2 son enfermedades crónicas y altamente prevalentes, las cuales con frecuencia concurren en un mismo paciente ${ }^{1,2}$. Desde los primeros estudios epidemiológicos que se desarrollaron en pro de dilucidar la génesis e historia natural de las 
enfermedades cardiovasculares (tales como el estudio de Framingham), la diabetes mellitus tipo 2 se ha reconocido como un factor de riesgo para el desarrollo de insuficiencia cardiaca. Dicho efecto aumenta de manera proporcional conforme mayor sea la afectación metabólica ${ }^{2,3}$. De igual manera, tanto el pronóstico como la respuesta al manejo farmacológico de la falla cardíaca varían en el paciente diabético, pero es mayor la morbimortalidad en quienes coexisten ambas ${ }^{4,5}$.

Por otro lado, múltiples estudios han puesto en evidencia la naturaleza bidireccional de este fenómeno; la insuficiencia cardiaca es un factor de riesgo para el desarrollo de resistencia a la insulina y diabetes mellitus tipo $2^{6,7}$. Este aumento del riesgo de sufrir diabetes en pacientes con falla cardiaca fue ilustrado en análisis subsecuentes de los estudios CHARM (Candesartan in Heart Failure Assesment of Reduction in Mortality and Morbidity) y EMPHASIS-HF (Eplerenone in Mild Patients Hospitalization and Survival Study in Heart Failure), en los que se evidenció una incidencia de diabetes mellitus tipo 2 marcadamente superior a la esperada $^{8,9}$.

De igual modo, un estudio basado en datos del Registro Nacional de Pacientes de Dinamarca evidenció que la severidad de la falla cardiaca medida en términos de la dosis de diuréticos de asa requeridos, predice la aparición de diabetes mellitus posterior a un infarto agudo de miocardio ${ }^{10}$. Por último, un análisis subsecuente de datos recogidos de esta cohorte danesa arrojó dos conclusiones interesantes: en primer lugar, se evidenció que la incidencia de diabetes mellitus tipo 2 de novo en pacientes con falla cardiaca se aproxima al $2 \%$ en el primer año después del diagnóstico, y se incrementa progresivamente hasta alcanzar un $3 \%$ después de cinco años de realizado el diagnóstico ${ }^{11}$. En segundo lugar, se demostró que quienes desarrollaron diabetes mellitus tipo 2 de novo tuvieron un aumento significativo de la mortalidad por cualquier causa, en comparación con pacientes sin diabetes ${ }^{11}$.

La relación entre falla cardiaca y resistencia a la insulina ha sido igualmente estudiada y reconocida. Estudios realizados en la década de los noventa, en los que se utilizó la pinza hiperinsulinémica-euglicémica, dieron los primeros indicios de la existencia de hiperglicemia en ayunas y resistencia a la insulina en pacientes con falla cardiaca independientemente de su etiología ${ }^{12}$. Estudios más recientes han demostrado la alta prevalencia de trastornos en el metabolismo de los carbohidratos en pacientes con falla cardiaca. Por ejemplo, un estudio conducido a finales de la primera década del 2000 demostró en una población de 94 pacientes con falla cardiaca, una prevalencia del $15 \%$ de diabetes no conocida previamente, del $24 \%$ de intolerancia a la glucosa oral y del $27 \%$ de resistencia a la insulina, definida como un índice HOMA-IR (homeostasis model assessment-insulin resistance) mayor a $2^{13}$. Así, la existencia de alteraciones en el metabolismo de los carbohidratos se demostró en un $94,6 \%$ de los pacientes seleccionados ${ }^{13}$.

Ahora bien, aunque ya se conocen con claridad la existencia e impacto de esta relación, sus mecanismos fisiopatológicos han sido pobremente estudiados y aun no hay certeza de sus características. A continuación se revisarán los mecanismos que se han propuesto en el desarrollo de la diabetes mellitus tipo 2 en pacientes con insuficiencia cardiaca.

\section{Mecanismos de desarrollo de diabetes en pacientes con insuficiencia cardiaca}

Un número importante de propuestas científicas han surgido en torno a la asociación entre falla cardiaca, alteraciones metabólicas, resistencia a la insulina y diabetes mellitus ${ }^{14}$. En general, todas las aproximaciones científicas que se han acuñado a lo largo del tiempo para explicar este fenómeno no son mutuamente excluyentes y, por el contrario, es posible que en una aproximación ecléctica que permita la construcción de un modelo explicativo dinámico y sinérgico, se encuentre la explicación más adecuada del rol causal de la falla cardiaca en la génesis de la diabetes mellitus tipo 2. En todo caso, el pilar central de todos los modelos explicativos fisiopatológicos, parte de una relación inicial entre la falla cardiaca y una progresiva resistencia a la insulina, la cual, a la larga, degenerará en diabetes mellitus tipo 2.

Así, entre las teorías que se han desarrollado para este fin, se destacan las siguientes:

- Activación del sistema neurohumoral: el sistema reninaangiotensina-aldosterona y el sistema nervioso autónomo simpático son protagonistas para entender la relación entre falla cardiaca y resistencia a la insulina ${ }^{15,16}$.

- Inflamación sistémica secundaria a un estado basal de hipoperfusión orgánica, evidenciada por un aumento significativo en los niveles plasmáticos de mediadores inflamatorios solubles y citoquinas tales como el factor de necrosis tumoral alfa ${ }^{17,18}$. Estos mediadores proinflamatorios disminuyen la expresión de los receptores GLUT 4, al alterar la cascada de fosforilación mediada por el receptor de insulina (receptor tipo tirosina quinasa) y su sustrato (insulin receptor substrate) ${ }^{18}$.

- Disminución progresiva de la función y masa total del músculo esquelético como resultado de la intolerancia al ejercicio y reducción de la actividad física ${ }^{14}$.

- Disminución de la perfusión hacia el páncreas y el músculo esquelético, que lleva a una disminución progresiva en la producción de insulina, al igual que del aporte y la captación de glucosa en los tejidos periféricos ${ }^{19}$.

- Aumento de adipocinas tales como adiponectina y leptina ${ }^{20}$.

- Exacerbación farmacológica de la resistencia a la insulina secundaria al uso de diuréticos y otros medicamentos.

De los anteriores modelos explicativos, el primero es el que ha recibido mayor atención y el que en este momento tal vez tenga evidencia más robusta de ser el responsable de las alteraciones metabólicas en los pacientes con falla cardiaca. En el contexto de una depresión del gasto cardiaco y subsecuentemente de la perfusión tisular, existe una homeostasis anómala y contraproducente del sistema humoral, a expensas de una activación permanente del sistema nervioso autónomo simpático y del eje renina-angiotensinaaldosterona $^{21}$. Es sabido que la activación permanente del sistema nervioso simpático es, en extremo, perjudicial para el miocardio, ya que genera un desbalance en la relación aporte-consumo de oxígeno, promoviendo hipoxia y muerte celular ${ }^{22}$.

En cuanto a la génesis de la diabetes mellitus tipo 2 en el marco de una activación simpática excesiva se ha 
demostrado que las concentraciones de norepinefrina en sangre se correlacionan tanto con los parámetros hemodinámicos de la falla cardiaca, como con la sensibilidad a la insulina y la tolerancia a la glucosa ${ }^{1,19,23}$. Son múltiples las formas mediante las cuales la activación del sistema nervioso simpático lleva a una disminución en la sensibilidad a la insulina. En primer lugar, el agonismo simpático sobre los receptores beta a nivel del adipocito aumenta la lipólisis del tejido graso y, por ende, los niveles de ácidos grasos libres circulantes ${ }^{24}$. Algunos estudios han avalado esta hipótesis, demostrando que la activación simpática es responsable del aumento en los niveles de ácidos grasos libres en pacientes con falla cardiaca, al activar la lipólisis mediante la activación de la lipasa sensible a hormonas ${ }^{25}$. De manera recíproca, los niveles de ácidos grasos en sangre se asocian de forma directamente proporcional a los niveles de catecolaminas circulantes, creando un bucle de activación que perpetúa este fenómeno ${ }^{26}$.

En el músculo esquelético de los humanos existe una relación dosis-respuesta entre los niveles plasmáticos de ácidos grasos y alteraciones en la cascada de señalización de la insulina ${ }^{27}$. Lo anterior se ha explicado por la activación mediada por ácidos grasos de la proteína kinasa $c$ (PKC), la cual fosforila el receptor de insulina, disminuyendo la translocación a la membrana plasmática con una consecuente reducción del importe de glucosa en el músculo ${ }^{28}$. Así, disminuciones agudas en los niveles plasmáticos de ácidos grasos aumentan la captación de glucosa por los tejidos periféricos e incrementan la sensibilidad a la insulina, independientemente del estado basal ${ }^{25}$. Aunado a lo anterior, los incrementos en los niveles circulantes de ácidos grasos se relacionan con un aumento en los niveles de triglicéridos, metabolitos de ácidos grasos en el citoplasma (tales como el diacilglicerol, el cual tiene una función clave en múltiples cascadas de señalización intracelulares) y activación de la proteína quinasa $C$ y el factor nuclear Kappa-B. Este último cumple un rol clave en las cascadas de señalización del sistema inmune y en la propagación de los estados proinflamatorios, promoviendo indirectamente la resistencia a la insulina, al aumentar los niveles circulantes del factor de necrosis tumoral alfa ${ }^{29}$.

Adicionalmente, el aumento en los niveles plasmáticos de catecolaminas y cortisol se asocia a un incremento en la glicemia ${ }^{30}$. La activación del sistema simpático y la liberación de cortisol (fisiológicamente dada como una respuesta al estrés o la hambruna), estimula y activa la glicógenofosforilasa hepática (enzima encargada de la glicogenólisis), así como varias enzimas necesarias para la gliconeogénesis en el hígado ${ }^{31,32}$. El resultado final de ambos procesos es un incremento sostenido en la glicemia.

El rol del eje renina-angiotensina-aldosterona en la génesis de la diabetes viene de los múltiples ensayos clínicos que confirmaron el uso de los inhibidores de la enzima convertidora de angiotensina (IECA), los antagonistas del receptor de angiotensina II (ARA-II) y los antagonistas de mineralocorticoides (AMC) en falla cardiaca e hipertensión, y aunque el diseño experimental de estos estudios no estuvo concebido para tal fin, varios de ellos demostraron que tanto los IECA como los ARA-II, redujeron significativamente la incidencia de diabetes mellitus tipo 2. Estudios como el HOPE
(Heart Outcomes Prevention Evaluation) con ramipril ${ }^{33}$, el CAPPP (Captopril Prevention Project) con captopril ${ }^{34}$ y el LIFE (Losartan Intervention for Endpoint Reduction in Hypertension) con losartán ${ }^{35}$, sugirieron, de manera consistente e inesperada, una reducción en la incidencia de diabetes de novo en el grupo de pacientes asignados a estos medicamentos ${ }^{36}$.

Por otro lado, estudios preclínicos han sugerido un efecto directo de la angiotensina II y de la activación de los receptores de mineralocorticoides por parte de la aldosterona, en la alteración tanto en la sensibilidad de los tejidos periféricos como en la secreción de insulina ${ }^{37,38}$. Tanto la angiotensina-II como la aldosterona inhiben la translocación mediada por la insulina de los receptores GLUT-4, disminuyendo la captación periférica de glucosa y aumentando la resistencia a la insulina ${ }^{37}$. De manera similar, ambas moléculas alteran la función de las células beta del páncreas, cambiando el umbral de glucosa al cual se inicia la liberación de insulina ${ }^{34,39,40}$. Como resultado, la activación excesiva del eje renina-angiotensina-aldosterona es una posible explicación para la resistencia a la insulina y la diabetes mellitus tipo 2 en los pacientes con insuficiencia cardiaca.

Por último se explorará la relación existente entre los agentes modificadores de la enfermedad (específicamente los betabloqueadores) y el desarrollo de resistencia a la insulina. El efecto de los betabloqueadores en el metabolismo de los carbohidratos y lípidos depende, en gran medida, de su generación y especificidad sobre los receptores adrenérgicos ${ }^{1}$. De esta manera, múltiples estudios evidenciaron que los betabloqueadores de primera generación aumentaban de manera considerable el riesgo de desarrollar diabetes mellitus tipo 2. El estudio ARIC (Atherosclerosis Risk in Communities) evidenció que los pacientes medicados, ya fuera con timolol o propanolol, tenían un $28 \%$ más riesgo de desarrollar diabetes (riesgo relativo 1,28, IC $95 \% 1,04-1,57)^{41}$.

En cuanto a los betabloqueadores de segunda generación, un estudio demostró un efecto menor del metoprolol y del atenolol sobre la resistencia a la insulina, con una disminución en esta del 13 y el $20 \%$, respectivamente ${ }^{42}$. Por último, los betabloqueadores de tercera generación, como el carvedilol, ejercen antagonismo no selectivo de los receptores beta y alfa, hecho que promueve un efecto diferente sobre el metabolismo de los glúcidos ${ }^{43}$. Un estudio que incluyó 72 pacientes a quienes se les evaluó el metabolismo del carvedilol vs. metoprolol en no diabéticos, demostró una disminución del $14 \%$ en la sensibilidad a la insulina en aquellos que recibieron metoprolol frente a un aumento cercano al $9 \%$ en aquellos que recibieron carvedilol ${ }^{44}$. Por último, un análisis subsecuente del estudio COMET (Carvedilol or Metoprolol European Trial), evidenció una diferencia significativa en la incidencia de diabetes de novo entre ambos medicamentos, siendo el carvedilol un factor protector para el desarrollo de esta patología (riesgo relativo=0,78; IC 95\% $0,61-0,99$, p 0,048$)^{45}$.

\section{Conflicto de intereses}

Ninguno. 


\section{Bibliografía}

1. Flores-Le-Roux JA, Benaiges Boix D, Pedro-Botet J. Insuficiencia cardíaca y desarrollo de diabetes mellitus tipo 2. Med Clin (Barc). 2012;138:579-83.

2. Kenny HC, Abel ED. Heart Failure in type 2 diabetes mellitus. Circ Res. 2019;124:121-41.

3. Kannel WB, Hjortland M, Castelli WP. Role of diabetes in congestive heart failure: The Framingham study. Am J Cardiol. 1974;34:29-34.

4. Bauters C, Lamblin N, Mc Fadden EP, Van Belle E, Millaire A, de Groote P. Influence of diabetes mellitus on heart failure risk and outcome. Cardiovasc Diabetol. 2003;2:1.

5. de Groote P. Impact of diabetes mellitus on long-term survival in patients with congestive heart failure. Eur Heart J. 2004;25:656-62.

6. Campbell P, Krim S, Ventura H. The bi-directional impact of two chronic illnesses: heart failure and diabetes \&\#150; a review of the epidemiology and outcomes. Card Fail Rev. 2015;1:8.

7. Guglin M, Lynch K, Krischer J. Heart failure as a risk factor for diabetes mellitus. Cardiol. 2014;129:84-92.

8. Preiss D, Zetterstrand S, McMurray JJV, Östergren J, Michelson EL, Granger CB, et al. Predictors of development of diabetes in patients with chronic heart failure in the Candesartan in Heart Failure Assessment of Reduction in Mortality and Morbidity (CHARM) program. Diabetes Care. 2009;1:915-20.

9. Preiss D, Van Veldhuisen DJ, Sattar N, Krum H, Swedberg K, Shi $\mathrm{H}$, et al. Eplerenone and new-onset diabetes in patients with mild heart failure: Results from the Eplerenone in Mild Patients Hospitalization and Survival Study in Heart Failure (EMPHASISHF). Eur J Heart Fail. 2012;14:909-15.

10. Andersson C, Norgaard ML, Hansen PR, Fosbøl EL, Schmiegelow $M$, Weeke $P$, et al. Heart failure severity, as determined by loop diuretic dosages, predicts the risk of developing diabetes after myocardial infarction: A nationwide cohort study. Eur J Heart Fail. 2010;12:1333-8.

11. Zareini B, Rørth R, Holt A, Mogensen UM, Selmer C, Gislason $G$, et al. Heart failure and the prognostic impact and incidence of new-onset of diabetes mellitus: A nationwide cohort study. Cardiovasc Diabetol. 2019;18:1-10.

12. Paolisso G, De Riu S, Marrazzo G, Verza M, Varricchio $M$, D'Onofrio F. Insulin resistance and hyperinsulinemia in patients with chronic congestive heart failure. Metabolism. 1991;40:972-7.

13. Clodi M, Resl M, Stelzeneder D, Pacini G, Tura A, Mörtl D, et al. Interactions of glucose metabolism and chronic heart failure. Exp Clin Endocrinol Diabetes. 2008;117:99-106.

14. Ashrafian H, Frenneaux MP, Opie LH. Metabolic mechanisms in heart failure. Circulation. 2007;116:434-48.

15. Kostis JB, Sanders M. The association of heart failure with insulin resistance and the development of type 2 diabetes. Am J Hypertens. 2005;18:731-7.

16. Grundy SM. Does the metabolic syndrome exist? Diabetes Care. 2006;29:1689-92.

17. Wisniacki N. Insulin resistance and inflammatory activation in older patients with systolic and diastolic heart failure. Heart. 2005;91:32-7.

18. Akash MSH, Rehman K, Liaqat A. Tumor necrosis factor-alpha: role in development of insulin resistance and pathogenesis of type 2 diabetes mellitus. J Cell Biochem. 2018;119: 105-10.

19. Guglin M, Villafranca A, Morrison A. Cardiogenic diabetes. Heart Fail Rev. 2014;19:595-602.

20. Doehner W, Rauchhaus M, Godsland IF, Egerer K, Niebauer J, Sharma $\mathrm{R}$, et al. Insulin resistance in moderate chronic heart failure is related to hyperleptinaemia, but not to norepinephrine or TNF-alpha. Int J Cardiol. 2002;83:73-81.
21. Zucker IH. Novel mechanisms of sympathetic regulation in chronic heart failure. Hypertension. 2006;48:1005-11.

22. Opie L. Adrenaline-induced "oxygen-wastage" and enzyme release from working rat heart. Effects of calcium antagonism, $\beta$-blockade, nicotinic acid and coronary artery ligation. J Mol Cell Cardiol. 1979;11:1073-94.

23. Levine TB, Francis GS, Goldsmith SR, Simon AB, Cohn JN. Activity of the sympathetic nervous system and renin-angiotensin system assessed by plasma hormone levels and their relation to hemodynamic abnormalities in congestive heart failure. Am J Cardiol. 1982;49:1659-66.

24. Ng TB. Adrenergic control of lipolysis in adipocytes of several mammalian species. Comp Biochem Physiol Part C Comp Pharmacol. 1985;82:463-6.

25. Marangou AG, Alford FP, Ward G, Liskaser F, Aitken PM, Weber $\mathrm{KM}$, et al. Hormonal effects of norepinephrine on acute glucose disposal in humans: A minimal model analysis. Metabolism. 1988;37:885-91.

26. Paolisso G, Manzella D, Rizzo MR, Ragno E, Barbieri M, Varrichio G. Elevated plasma fatty acid concentrations stimulate the cardiac autonomic nervous system in healthy subjects. Am J Clin Nutr. 2000;72:723-30.

27. Belfort R, Mandarino L, Kashyap S, Wirfel K, Pratipanawatr T, Berria R, et al. Dose-response effect of elevated plasma free fatty acid on insulin signaling. Diabetes. 2005;54:1640-8.

28. Itani SI, Ruderman NB, Schmieder F, Boden G. Lipid-induced insulin resistance in human muscle is associated with changes in diacylglycerol, protein kinase C, and I B-. Diabetes. 2002;51:2005-11.

29. Savage DB, Petersen KF, Shulman Gl. Mechanisms of insulin resistance in humans and possible links with inflammation. Hypertension. 2005;45:828-33.

30. Benedict CR, Weiner DH, Johnstone DE, Bourassa MG, Ghali $\mathrm{JK}$, Nicklas J, et al. Comparative neurohormonal responses in patients with preserved and impaired left ventricular ejection fraction: Results of the studies of left ventricular dysfunctions (SOLVD) registry. J Am Coll Cardiol. 1993;22:A146-53.

31. Han C, Rice MW, Cai D. Neuroinflammatory and autonomic mechanisms in diabetes and hypertension. Am J Physiol Metab. 2016;311:E32-41.

32. Nonogaki K. New insights into sympathetic regulation of glucose and fat metabolism. Diabetologia. 2000;43:533-49.

33. Heart Outcomes Prevention Evaluation (HOPE) Study Investigators. Effects of ramipril on cardiovascular and microvascular outcomes in people with diabetes mellitus: results of the HOPE study and Minsuficiencia cardiacaRO-HOPE substudy. Lancet. 2000;355(9200):253-9.

34. Hansson L, Lindholm LH, Niskanen L, Lanke J, Hedner T, Niklason $A$, et al. Effect of angiotensin-converting-enzyme inhibition compared with conventional therapy on cardiovascular morbidity and mortality in hypertension: the Captopril Prevention Project (CAPPP) randomised trial. Lancet. 1999;353:611-6.

35. Lindholm LH, Ibsen H, Dahlöf B, Devereux RB, Beevers G, de Faire $U$, et al. Cardiovascular morbidity and mortality in patients with diabetes in the Losartan Intervention For Endpoint reduction in hypertension study (LIFE): a randomised trial against atenolol. Lancet. 2002;359:1004-10.

36. Lim HS, MacFadyen RJ, Lip GYH. Diabetes mellitus, the reninangiotensin-aldosterone system, and the heart. Arch Intern Med. 2004; 164:1737.

37. Luther JM, Brown NJ. The renin-angiotensin-aldosterone system and glucose homeostasis. Trends Pharmacol Sci. 2011;32:734-9.

38. Joseph JJ, Echouffo Tcheugui JB, Effoe VS, Hsueh WA, Allison MA, Golden SH. Renin-Angiotensin-Aldosterone System, Glucose Metabolism and Incident Type 2 Diabetes Mellitus: MESA. J Am Heart Assoc. 2018;7:e009890. 
39. Underwood PC, Adler GK. The renin angiotensin aldosterone system and insulin resistance in humans. Curr Hypertens Rep. 2013;15:59-70.

40. Luther JM. Effects of aldosterone on insulin sensitivity and secretion. Steroids. 2014;91:54-60.

41. Gress TW, Nieto FJ, Shahar E, Wofford MR, Brancati FL. Hypertension and antihypertensive therapy as risk factors for type 2 diabetes mellitus. N Engl J Med. 2000;342:905-12.

42. Pollare $T$, Lithell $H$, Selinus I, Berne $C$. Sensitivity to insulin during treatment with atenolol and metoprolol: a randomised, double blind study of effects on carbohydrate and lipoprotein metabolism in hypertensive patients. BMJ. 1989;298: 1152-7.
43. Ripley TL, Saseen JJ. $\beta$-blockers: a review of their pharmacological and physiological diversity in hypertension. Ann Pharmacother [Internet]. 2014;48:723-33. Disponible en http://www.ncbi.nlm.nih.gov/pubmed/24687542.

44. Jacob S, Rett K, Wicklmayr M, Agrawal B, Augustin HJ, Dietze GJ. Differential effect of chronic treatment with two betablocking agents on insulin sensitivity: the carvedilol-metoprolol study. J Hypertens. 1996;14:489-94.

45. Torp-Pedersen C, Metra M, Charlesworth A, Spark P, Lukas MA, Poole-Wilson PA, et al. Effects of metoprolol and carvedilol on pre-existing and new onset diabetes in patients with chronic heart failure: data from the Carvedilol Or Metoprolol European Trial (COMET). Heart. 2007;93:968-73. 